\title{
LA EXPERIENCIA ESTÉTICA Y LOS ESTRATOS DE LA OBRA DE ARTE. LA ESTÉTICA COMO LA ESENCIA DEL ARTE
}

\section{The Aesthetic Experience and the Layers of the ARt Work. Aesthetics AS the essence Of ART}

\author{
Pau Pedragosa \\ Societat Catalana de Filosofia/ \\ Universitat Politècnica de Catalunya, España \\ pau.pedragosa@upc.edu
}

\begin{abstract}
Resumen: El contenido de este artículo consiste en mostrar que la experiencia estética es la esencia de la experiencia de la obra de arte. Argumentaré en contra de la concepción del arte de Arthur C. Danto según la cual el arte moderno ya no requiere de la experiencia estética y este hecho determina el fin del arte. La experiencia estética permitiría dar cuenta del arte desde el Renacimiento hasta el siglo XIX pero el arte moderno del siglo XX solo puede ser explicado conceptualmente $y$, por tanto, la filosofía del arte es necesaria para explicitar ese contenido.

Para defender el estatuto estético de la obra de arte mostraré que la experiencia estética se identifica con la experiencia fenomenológica. Esto quiere decir que la experiencia estética nos hace concientes de la diferencia entre el contenido de la obra (lo que aparece ) y el medio de la experiencia sensible en el que este contenido se da (el aparecer). El "aparecer" y "lo que aparece" se corresponden en la experiencia estética con los dos polos de la relación intencional y constituyen los dos estratos fundamentales de la obra de arte. A través de la aproximación fenomenológica intentaré mostrar que la obra de arte no excluye el contenido conceptual, pero este contenido ha de estar necesariamente incorporado. No es la filosofía la que tiene que comprender este contenido sino exclusivamente la experiencia estética.
\end{abstract}

Palabras clave: Estética, fenomenología, Husserl, Arthur C. Danto, impresionismo, arte abstracto, fin del arte.

\begin{abstract}
The subject of this paper is to claim that the aesthetic experience is the essence of the experience of the work of art. I argue against the view hold by Arthur C. Danto, according to which modern art does not require the aesthetic experience any more and that this fact means the end of art. The aesthetic experience allows explaining only the art made between the Renaissance and the XIX century. The modern work of art of the XX century can only be explained conceptually and therefore a philosophy of art is required to make that content explicit and clear.

To defend the aesthetic status of the work of art I will show that the aesthetic experience identifies itself with the phenomenological experience. This means that the aesthetic experience makes us aware of the difference between the content of the work (what appears) and the sensible lived experience in which this content appears (the appearance). The "appearance" and "what appears" are the two poles of Intentionality and the two fundamental layers of the work of art. Through the phenomenological approach I will make clear that the work of art does not exclude the conceptual content at all. This content has to be necessarily embodied. It is not philosophy that has to disclose this content but the aesthetic experience alone.
\end{abstract}

Key Words: Aesthetics, Phenomenology, Husserl, Arthur C. Danto, Impressionism, Abstract Art, End of Art. 


\section{LA TESIS DEL FIN DEL ARTE}

El arte abstracto como el suprematismo de Malevich y el neoplasticismo de Mondrian, así como otros tipos de obras de arte de Vanguardia como el readymade, ya sea Fountain (1917) de Marcel Duchamp o Brillo Box (1964), de Andy Warhol, han puesto de manifiesto, según la mayoría de críticos de arte, que muchas corrientes del arte moderno del siglo XX rechazan cualquier aproximación estética, en el sentido de que el contacto de la obra con los sentidos es insuficiente para dar cuenta de la obra. Quien con más insistencia ha defendido esta posición es Arthur C. Danto, el cual dice, por ejemplo, que "las obras de arte y las cosas reales no pueden ser distinguidas solo a través del reconocimiento visual" ${ }^{\prime 1}$. Danto identifica esta insuficiencia de la percepción sensible para dar cuenta de las obras de arte modernas como el acontecimiento que da lugar al fin del arte. Danto sigue explícitamente la famosa sentencia de Hegel:

[E]l arte, por lo que se refiere a su destino supremo, es y permanece para nosotros una cosa del pasado. Con ello, también ha perdido para nosotros la auténtica verdad y vitalidad. Si antes afirmaba su necesidad en la realidad y ocupaba el lugar supremo de la misma, ahora se ha desplazado más bien a nuestra representación. Lo que ahora despierta en nosotros la obra de arte es el disfrute inmediato y a la vez nuestro juicio, por cuanto corremos a estudiar (i) el contenido, (ii) los medios de representación de la obra de arte y la adecuación o inadecuación entre estos dos polos. Por eso, el arte como filosofía es más necesario en nuestro tiempo que cuando el arte como tal producía ya una satisfacción plena. El arte nos invita a la contemplación reflexiva, pero no con el fin de producir nuevamente arte, sino para conocer filosóficamente lo que es el arte. ${ }^{2}$

Según Hegel, cuando (i) el contenido (ideal, conceptual) del arte y (ii) el medio de presentación sensible se separan - cuando se someten a nuestro juicio para evaluar su mutua adecuación-, entonces se decreta el fin del arte tal y como había sido hasta entonces. Hegel piensa especialmente en el arte antiguo en el que se daba, sin problematizar, una manifestación sensible de la idea,

\footnotetext{
${ }^{1}$ Danto, Arthur C. After The End of Art. Contemporary Art and the Pale of History. Princeton University Press. New Jersey, 1997, p. 71

2 Citado per Danto en Op. Cit., p 31. Nota: Danto traduce por "filosofía" el término "Wissenschaft" de las Lecciones sobre Estética de Hegel.
} 
una adecuación entre el contenido y el medio de su presentación sensible. Cuando la idea ya no se puede manifestar sensiblemente o cuando el arte problematiza que pueda haber tal manifestación adecuada de la idea, entonces el arte debe ser explicado filosóficamente.

Danto comparte esta teoría del arte de Hegel. Lo que caracteriza al arte moderno es que su contenido está por encima de su aparecer sensible. Las obras encarnan ideas que son invisibles en la obra, son diferentes del aspecto que se muestra a los sentidos: "Hay una característica del arte contemporáneo que lo distingue de todo el arte desde 1400, y es que sus ambiciones principales ya no son estéticas" ${ }^{3}$. Por lo tanto: "la conexión entre arte y estética es una cuestión de contingencia histórica, y no una parte de la esencia del arte" ${ }^{\prime 4}$. La sentencia del fin del arte de Danto es básicamente hegeliana: "Pienso que el fin del arte consiste en el hacerse conciente de la naturaleza filosófica del arte. Este pensamiento es completamente hegeliano" ${ }^{5}$.

Según Danto la sentencia hegeliana no quedaría confirmada hasta 1964, cuando Warhol expone las Brillo Boxes en la Stable Gallery de Nueva York. El período histórico que es testigo del arte producido después de Hegel, entre 1828, cuando lee sus Lecciones sobre Estética y 1964, se puede caracterizar como un período intermedio, Ilamado por Danto la "Era de los Manifiestos" ${ }^{\prime 6}$. Las obras de arte de este período cuestionaron qué era esencialmente el arte y así expresaron diferentes ideas de qué es el arte. Estos años vendrían caracterizados por un conflicto entre diferentes filosofías del arte expuestas tanto en las mismas obras de arte como en los respectivos manifiestos que explicarían con claridad la filosofía del arte correspondiente, así como también por los críticos de arte, los comisarios de los museos, etc. (el llamado Artworld). La tarea de estas filosofías del arte fue durante este tiempo la de descubrir la esencia del arte, de manera que se estableció un conflicto dogmático entre excluyentes concepciones del arte verdadero. Durante esta época un movimiento artístico y un manifiesto se erigían como el único arte verdadero. Este período de conflicto de manifiestos acabaría precisamente en los alrededores de la década de 1960, en concreto el año 1964 con la obra de Warhol' .

\footnotetext{
3 Íbid., p. 183

4 Íbid., p 25

5 ïbid., p. 30

${ }^{6}$ Íbid., pp. 28-37

7 Ibid., pp 35, 37.
} 
Es entonces cuando, siempre según Danto, se alcanza otro nivel que supera la Era de los Manifiestos. Esta nueva fase del arte consiste en el descubrimiento de que no hay un arte más verdadero que otro, que no hay una manera en que el arte debe ser. La cuestión ahora es qué es arte en general ${ }^{8}$. Este descubrimiento lo hizo Warhol:

Desde mi punto de vista, la cuestión de qué es real y esencialmente el arte -a diferencia de lo que aparente o inesencialmente es- era la forma incorrecta de plantear la cuestión filosófica. [...]. Como yo lo veía, la forma de la cuestión es: ¿Qué diferencia hay entre una obra de arte y algo que no es una obra de arte cuando no hay diferencias perceptivas interesantes entre ambas? [...] El problema filosófico es ahora explicar por qué hay obras de arte. Con Warhol se vio claro que no hay una manera determinada de cómo debe ser una obra de arte - puede parecerse a una Brillo Box o puede parecerse a una lata de sopa. Warhol forma parte del grup de artistas que han hecho este descubrimiento profundo. La distinción entre música y ruido, entre danza y movimiento, entre literatura y mera escritura, fueron coetáneos de la irrupción de Warhol. ${ }^{9}$

Para Danto, pues, la problemática del arte contemporáneo se resume en la pregunta: "¿Qué diferencia hay entre una obra de arte y algo que no lo es cuando no hay diferencias perceptivas interesantes entre ambos?"10. El hecho de que los objetos de arte y los que no lo son sean indiscernibles desde el punto de vista de la sensibilidad implica tener que recurrir a la filosofía para saber cuándo estamos ante una obra de arte:

En relación a las apariencias, cualquier cosa podía ser una obra de arte, y eso significó que si querías descubrir qué era arte, tenías que hacer un giro de la experiencia sensible al pensamiento. Dicho brevemente, tenías que darte la vuelta hacia la filosofía. ${ }^{11}$

Las obras plantean estas cuestiones filosóficas: ¿Qué es arte? ¿Qué diferencia hay entre arte y no arte? La filosofía del arte sustituye a la experiencia estética y se realiza finalmente la tesis hegeliana del fin del arte.

Sin embargo, este tipo de interpretación, que reclama una superación de la

\footnotetext{
8 Ibid., p. 34

9 Íbid., p. 35

10 O también: "las obras de arte y las cosas reales no pueden ser distinguidas solo a través del reconocimiento visual", Danto, A. Op. Cit., p. 71

${ }_{11}$ Íbid., p. 13
} 
experiencia estética para dar cuenta de gran parte del arte moderno y del contemporáneo, no reconoce, como dice claramente Martin Seel ${ }^{12}$, la especificidad de la experiencia estética e identifica simplemente "estético" con lo que es perceptible sensiblemente. La afirmación de Danto de que "las obras de arte y las cosas reales no pueden ser distinguidas solo a través del reconocimiento visual"13 es cierta si por "reconocimiento visual" entendemos solo la visión natural cotidiana con la que miramos unas cajas del supermercado. Con esta actitud ciertamente nada distingue la Brillo Box de una caja de detergente ${ }^{14}$. Lo que Danto no reconoce es que dos objetos de aspecto idéntico aparecen de manera diferente si los percibimos según la actitud cotidiana o si los contemplamos estéticamente. Danto, pues, no reconociendo la especificidad de la experiencia estética, puede afirmar su contingencia histórica, es decir, su final, pues con ello se reconoce correctamente que la percepción sensible cotidiana no es esencial a la recepción del arte moderno. Si, en cambio, reconocemos que la experiencia estética no es equiparable a la experiencia sensible habitual, debemos afirmar que la experiencia estética no es contingente a la recepción de la obra de arte, sino esencial y, por tanto, no ha llegado a su fin. A esta experiencia y su singularidad dedicamos el siguiente capítulo.

\section{LA EXPERIENCIA ESTÉTICA}

La experiencia estética no se limita solo al arte. En principio cualquier objeto de la percepción sensible puede convertirse en un objeto estético si adoptamos la actitud adecuada. La obra de arte ofrece un caso especialmente señalado de la percepción estética: aquél en el que se adoptará esta actitud para hacerle justicia. Por tanto, es en el marco de la percepción sensible en general donde debemos demarcar la percepción estética, de la que la percepción estética del arte es un caso particular. La percepción estética hay que entenderla, pues, principalmente, como parte de la percepción sensible en general, es aquí donde debe comenzar y no en el arte en particular.

La percepción sensible humana se caracteriza por la posibilidad de desple-

\footnotetext{
12 Seel, Martin. Estética del aparecer. Madrid: Katz editores. 2010, pp. 182-186 ("La objeción de Danto"). Seguiremos a este autor en algunas consideraciones importantes, especialmente en nuestro $\S 3$ y en las conclusiones

${ }^{13}$ Danto, A. Op. Cit., p. 71

${ }^{14}$ Cf. Seel, M. Op. Cit., p. 186
} 
garse en una experiencia articulada mediante conceptos ${ }^{15}$. Pero esta posibilidad no siempre hay que llevarla a cabo. Cuando este es el caso nos encontramos ante la percepción estética. Es, por tanto, en el marco de la experiencia conceptual que puede establecerse la particularidad de la experiencia estética. Ésta última presupone la capacidad de percibir cualquier cosa y determinarla conceptualmente ya que solo quien puede percibir conceptualmente puede prescindir de fijar esta determinación y entonces la podrá percibir en la plenitud de sus aspectos sensibles, en una presencia no sometida a determinaciones.

Paul Valéry nos ofrece una clara explicación de cómo procede la determinación perceptiva de un objeto ${ }^{16}$ : entre las múltiples impresiones visuales que muestran los objetos según desde donde los miramos, la mirada aísla solo unas cuantas que sabe interpretar, bien reconocibles y que sirven de referencia para identificar los objetos. Esta limitación de las impresiones se da por el criterio del hábito, la costumbre, que impone una construcción de la visión que nos hace ver mediante abreviaciones y sustituciones que la primera educación nos ha enseñado. Esta simplificación de la mirada permite que de las innumerables apariencias de las cosas solo una parte débil y muy pequeña sea relevante para nuestro vivir diario, de tal forma que percibimos de los objetos solo lo necesario para sustituirlos por un concepto, ya sea una comprensión o una acción determinadas. No perdemos tiempo en la percepción pues ésta se resuelve en algo diferente. En estos casos lo sensible de los objetos no se conserva o, como dice Valéry, no sobrevive a su comprensión una vez ha cumplido su papel, una vez ha realizado su función ${ }^{17}$.

Es importante darse cuenta de que no utilizar la abreviatura conceptual de la percepción no quiere decir que no hayan conceptos correspondientes, sino que significa que no nos interesa comprender solo conceptualmente. Entonces ponemos la atención en la presencia fenoménica del objeto. Esto quiere decir que saber qué objeto estoy percibiendo estéticamente no disminuye la intensidad estética de la percepción, simplemente pongo este conocimiento del objeto entre paréntesis. Es importante el hecho de que solo quien puede percibir conceptualmente puede decidir no hacer un uso de conceptos. La aparición sensible que la percepción estética revela no es anterior a la actividad conceptual,

\footnotetext{
${ }^{15}$ Cfr. Seel, M. Op. Cit., p. 48

${ }^{16}$ Valéry, Paul. "Poesía y pensamiento abstracto" en Teoría poética y estética. Madrid: Visor, 1990

17 "ha actuado; ha cumplido su función; ha hecho comprender: ha vivido" Valéry, P. Op. Cit., p. 85 
sino posterior, sé en cada momento que lo que aparece lo puedo captar también conceptualmente, pero también lo puedo percibir de tal forma que sea inagotable mediante conceptos. Por tanto, los seres vivos que, como los animales, tienen percepción sensible pero no la articulan conceptualmente, no pueden tener percepción estética.

Si en la percepción estética lo que aparece no está fijado conceptualmente, si no aparece como algo, entonces lo que hay es un aparecer de sí mismo, una presentación del aparecer o una exposición del mismo aparecer ${ }^{18}$. En este sentido podemos caracterizar la percepción estética como la apertura de un ámbito del aparecer o como la atención a un juego de apariciones sensibles, en el que lo que se muestra aparece de una manera diferente, que de otra manera sería inaccesible. En la experiencia estética no percibimos un mundo diferente del mundo de los objetos sensibles, sino este mismo mundo percibido de otra manera: con una intensidad del aquí y del ahora en el que tiene lugar la percepción.

Hay que decir también que la experiencia estética no es, obviamente, el único modo de experiencia del mundo ni tampoco el más importante. Si lo que se trata es de orientarnos en el mundo desde una perspectiva práctica, debemos conducirnos de otra manera, debemos fijar conceptualmente lo que se nos presenta. Aquí reside la diferencia entre la percepción estética respecto de cualquier orientación teórica o práctica hacia el mundo: la percepción estética es desinteresada, como dice Kant, o inútil, según Valéry. Pero aquí reside también su importancia: su sentido se encuentra exclusivamente en sí mismo y, así, permite alcanzar un contacto con el mundo en el que no interesa nada más que este contacto.

La percepción estética comienza ahí donde dejamos a las cosas ser tal como aparecen aquí y ahora. Esta es también la tarea de la fenomenología. La estética hay que entenderla como fenomenología, coincide con ella. La fenomenología consiste en el esfuerzo de buscar la intuición plena o la experiencia vivida concreta que se encuentra por debajo de toda abstracción y teoría elaborada. La praxis cotidiana impide constantemente realizar esta intuición directa porque, como hemos dicho, se da por satisfecha con mencionar las cosas me-

\footnotetext{
${ }^{18}$ Seel, M. Op. Cit., pp. 148, 256, 261
} 
diante las abreviaciones de signos, fórmulas, conceptos y palabras ${ }^{19}$. La vida cotidiana es muy abstracta y de lo que se trata es de ver la experiencia vivida que subyace a estas abstracciones.

Lo que en nuestra actitud natural de la vida cotidiana nos aparece es siempre algo determinado, esto o aquello, un ente, algo, pero no el medio a través del cual algo se nos presente. Husserl introduce la distinción entre "lo que aparece" y "el aparecer" o, con más exactitud, "los apareceres" o "las apariencias" $^{20}$. "Lo que aparece" es el objeto y "el aparecer" son las parcialidades, los aspectos múltiples, a través de los cuales percibimos el objeto. Solo tenemos acceso perceptivo al objeto "mediado por" o "a través de" ${ }^{21}$ los modos en que aparece. No hay objeto sin la mediación de esa pluralidad de aspectos en la que el objeto se da, no hay un atajo directo al objeto en sí mismo. El objeto se constituye dentro de esa relación de mediación que Husserl llama intencionalidad. Ser fiel a la cosa en sí misma significa atender con todo detalle a todos estos múltiples modos del aparecer.

Hay que mantener, pues, la diferencia entre el aparecer múltiple y lo que aparece; entre la percepción y lo que se percibe: el decir y lo dicho, el ver y lo visto, el oír y lo oído. Lo que en cada caso aparece en la actitud natural cotidiana es lo dicho, lo visto, lo oído, y lo que permanece oculto en esta misma actitud pero que la fenomenología debe hacer explícito es: el decir, el ver, el oír o, con más precisión: lo dicho en el decir, lo visto en el ver, lo oído en el oír.

\section{LOS ESTRATOS DE LA OBRA DE ARTE}

Esta diferencia fundamental entre el aparecer y lo que aparece, la tensión entre la percepción y lo percibido, es lo que nos permite establecer diferentes estratos en la obra de arte $\mathrm{e}^{22}$ : el contenido de la obra y su medio de presenta-

\footnotetext{
${ }^{19}$ Blumenberg, Hans. "Mundo de la vida y tecnificación bajo los aspectos de la fenomenología" en Las realidades en que vivimos. Paidós. Barcelona. 1999, p. 43.

${ }^{20}$ Husserl, Edmund. Husserliana 21, 1983. Studien zur Arithmetik und Geometrie. Gesammelte Werke, Ed. Ingeborg Strohmeyer. Den Haag: Martinus Nijhoff, 1983, p. 282.

${ }^{21}$ Tani, Toru. "Appearences" en Handbook of Phenomenological Aesthetics. Edited by Hans Rainer Sepp, Lester Embree. Heidelberg London New York: Springer Dordrecht, 2010, p. 18.

${ }^{22}$ Esta diferencia es la que lleva a Roman Ingarden a considerar la obra de arte como una construcción estratificada. Ver: Ingarden, Roman. Ontology of the Work of Art. The Musical Work, The Picture, The Architectural Work, The Film. Ohio University Press. 1989 . Ver también: Escoubas, Eliane. "Painting" en Handbook of Phenomenological Aesthetics. Op. Cit., p 251. "Here painting would put into work the "how" (das Wie)-the eidos (aspect)-insofar as the aspect is no being, but the appearing of that which appears." Y Tani, Toru. "Appearences". Op. Cit., p. 18.
} 
ción. Son los dos estratos que con su separación, según Hegel, se llega al fin del arte tal como se entendía hasta entonces. Analizaremos a continuación los estratos de la obra pictórica - aunque este análisis se puede hacer con respecto a cualquier género artístico ${ }^{23}$ - y cómo se separan en el impresionismo y en la llamada pintura abstracta.

Aunque estos son los dos estratos fundamentales de la obra de arte, los estéticamente relevantes, podemos distinguir aún otro estrato previo, normalmente obviado. Este estrato no es otro que el soporte material de la obra. En su materialidad vemos un objeto de tela (pintura moderna), de madera (pintura antigua), de vidrio (vidrieras medievales), de piedra y vidrio (mosaicos), de cobre (grabados sobre planchas de cobre), etc. Este soporte material sobre el que se pinta el cuadro, así como las mismas manchas de pintura como material físico, es algo físico del mundo. Este primer estrato tiene una importancia relativa en la medida en que, por ejemplo, el brillo del cobre o el material de la madera colaboran con el tono del color de la pintura. Pero exceptuando esa sutileza, desde un punto de vista estético este estrato es el menos relevante, si bien es de enorme importancia en otros géneros artísticos como la arquitectura. También es importante si nuestra actitud no es estética, sino por ejemplo restauradora del cuadro.

Mucho más relevante es el segundo estrato, el que caracterizamos como "lo que aparece", lo representado o el contenido del cuadro: un paisaje, un bodegón, un retrato, un acontecimiento mítico, religioso o histórico. Efectivamente, cuando miramos un cuadro lo que de hecho vemos es lo que éste representa y no el soporte material. La realidad representada en el cuadro no es real en el sentido de la realidad material, es una realidad imaginada, una ilusión, pero sigue teniendo el sentido de objeto, pues vemos en el cuadro objetos como cuerpos humanos o animales, o cosas como edificios, muebles, árboles.

¿Cómo vemos estos objetos que aparecen sobre el cuadro? ¿Cuál es la forma de aparecer de estos objetos representados? Aquí es donde aparece el estrato más fundamental de todos, el que caracterizamos como "el aparecer": las parcialidades, los múltiples aspectos, a través de los cuales percibimos el objeto representado. Este estrato, que se encuentra entre los dos anteriores, consiste

\footnotetext{
${ }^{23}$ Ingarden analiza los estratos de las diferentes obras de arte pertenecientes a diferentes géneros artísticos: literatura, música, pintura, arquitectura, cine y teatro
} 
en las manchas de pintura repartidas por encima de la superficie material, pero no vistas como manchas de pintura en su materialidad como cosa física (estrato 1), sino interpretadas como la representación de una selección de los diferentes aspectos o apariencias del objeto que el artista quiere representar.

Según Husserl, todo objeto es un polo de identidad más allá de la multiplicidad de sus apariciones sensibles. Lo que es efectivamente dado a la percepción son los diferentes lados, perfiles, escorzos, en una palabra, los aspectos sensibles (Abschattungen ${ }^{24}$ ) "a través" de los cuales percibimos el objeto intencional. El objeto intencional y la pluralidad de aspectos sensibles "a través" del cual éste se presenta a la sensibilidad, son los dos polos de la relación intencional y pertenecen a dos órdenes distintos. El objeto hacia el que convergen sintéticamente los múltiples aspectos, no es un aspecto más ni la suma de todos ellos, sino que se encuentra a otro nivel. Estos dos niveles son los dos estratos fundamentales de la pintura. En estos mismos términos Roman Ingarden explica la pintura figurativa:

La actividad del pintor consiste - si bien no exclusivamente- en reconstruir en el cuadro, con los medios de la pintura, los aspectos visuales, perceptivos, apropiados, asumiendo que el cuadro se debe construir de tal manera que pueda llevar este $o$ aquel objeto a la presencia del observador. [...] Los aspectos forman el elemento constitutivo más importante del cuadro sin el cual no habría cuadro figurativo en absoluto. ${ }^{25}$

Podemos resumir los tres estratos de la obra pictórica figurativa del siguiente modo:

1. El soporte material sobre el que se extiende la pintura: desde el punto de vista estético, este estrato propiamente no lo vemos, la mirada no se dirige a un objeto físico colgado de la pared (que vemos en escorzo etc.), sino directamente a la representación pictórica. El soporte material es negado por las manchas de pintura y por lo que se representa a través de éstas.

2. La superficie pictórica: las manchas de pintura sobre una superficie de dos dimensiones vistas como los "aspectos reconstruidos" del objeto represen-

\footnotetext{
${ }^{24}$ Husserl, Edmund. Husserliana 3/1, Ideen zu einer reinen Phänomenologie und phänomenologischen Philosophie, Band I. Gesammelte Werke, Ed. Karl Schuhmann, Den Haag: Martinus Nijhoff, 1976, §42, p. 86-89. Husserl llama a las partes, perfiles o escorzos, "Abschattungen". Para un comentario de las "Abschattungen" ver: Sokolowski, Robert. 1974. Husserlian Meditations. How Words Present Things. Northwestern University Press. Evanston, 1974, p. 89-93.

${ }^{25}$ Ingarden, R. Op. Cit., pp. 149, 150
} 
tado. "El aparecer" "a través" del cual percibimos el objeto representado o "lo que aparece".

3. El objeto representado en el cuadro ${ }^{26}$ o "lo que aparece".

Por su irrelevancia desde el punto de vista estético, podemos prescindir del primer estrato y centrarnos en el segundo y el tercero, los realmente importantes para la percepción estética: el contenido (objeto o tema representado) y su medio de presentación. Ambos estratos equivalen en términos estéticos a los dos polos de la experiencia perceptiva: el objeto percibido y los múltiples aspectos a través de los cuales o con cuya mediación percibimos el objeto.

La mirada del observador recorre y se entretiene en las manchas de pintura extendidas sobre la superficie del cuadro y las reconoce inmediatamente como aspectos del objeto que aparece "a través" de ellas. A través de los aspectos sensibles reconstruidos por el artista, el observador los trasciende y ve el objeto representado en su totalidad. Por eso dice Ingarden que:

Los aspectos deben ser reconstruidos de tal forma que no llamen sobre ellos mismos la atención del observador, que sean en consecuencia simplemente vividos, ya que solo entonces pueden cumplir su función figurativa. ${ }^{27}$

En este sentido se ha entendido la pintura figurativa en perspectiva desde el Renacimiento como una ventana "a través" de la cual se ve un mundo imaginado, una ilusión ${ }^{28}$. Es decir, a través del segundo estrato (selección de los aspectos sensibles del objeto representado) se ve, como al otro lado de la ventana, el tercero (el objeto representado). Cuando con el impresionismo se debilitó el estrato del objeto representado, entonces el estrato de los aspectos sensibles se puso en primer término. Con el impresionismo se abrió paso el arte abstracto en un proceso según el cual los aspectos sensibles no debían ya ser reconstruidos de tal forma que no llamasen sobre ellos mismos la atención del observador - para que se pudiera ver "a través" suyo-, sino que, al contrario, retu-

\footnotetext{
${ }^{26}$ Ingarden añade un estrato más quando lo representado no es, por ejemplo, un bodegón, donde solo hay que ver las manchas de pintura como los "aspectos reconstruidos" de un objeto de la percepción para comprender el cuadro, sino quando hay representado un tema mítico, histórico o religioss, ya que entonces interviene un estrato superior de significación que permite comprender una narración más compleja que lo acerca a la literatura, por esta razón llama a este estrato "tema literario". Nosotros, para simplificar, juntamo estos dos estratos en uno: el objeto representado, ya sea éste una historia narrativa o bien un objeto de la percepción.

${ }^{27}$ Ingarden; R. Op. Cit., p. 150

28 Panofsky, E. La perspectiva como forma simbólica. Barcelona: Paidós. 2008 (3a ed.), p. 11.
} 
vieran sobre sí la atención, dificultando de este modo la percepción del objeto representado, obstaculizando el paso al tercer estrato. Con la apertura de este camino que lleva al arte moderno, los aspectos dejan de ser simplemente vividos para hacerse explícitamente vividos; el tercer estrato se debilita a favor del segundo; el objeto representado se debilita a favor de sus múltiples aspectos sensibles. Como se suele decir: el arte se hizo conciente de sí mismo y descubrió lo que es esencial al arte: la experiencia estética y el medio del aparecer. El arte desde entonces nos hace concientes de la diferencia entre el objeto representado y la pluralidad de sus manifestaciones o el juego de sus apariciones sensibles. Solo entonces el ámbito del aparecer se hace temático.

\section{LA TEMATIZACIÓN DE LOS ESTRATOS EN EL IMPRESIONISMO Y LA ABSTRACCIÓN}

Con el impresionismo comienza el proceso que elimina la ilusión o la representación del objeto y se afirma la pintura como una superficie de impresiones visuales, una superficie de aspectos sensibles que el observador se ve incapaz de sintetizar y trascender en un objeto representado. Esta tensión entre lo percibido y la percepción, entre un mundo representado y la superficie del cuadro que contiene manchas de colores fue la gran aportación del impresionismo. La mirada del observador llega a captar un objeto representado pero no se la deja proseguir con naturalidad hasta identificarlo inequívocamente porque la mirada es frenada en la superficie, en la que se recrea observando los diferentes colores y formas en sí mismas, sin poder reconocerlas como aspectos de un objeto de la percepción: este color como la propiedad de un objeto, esa forma como un escorzo visual, etc. La superficie del cuadro no abre el paso a la representación de un mundo. Aparece así una tensión entre los dos estratos, entre la superficie pictórica y el objeto representado. Esta tensión es correlativa a la que tiene lugar en la experiencia del observador que entonces se hace explícitamente estética: en un primer momento la percepción intenta identificar objetos, intenta trascender la percepción, a través de los aspectos sensibles, hacia la ilusión de la realidad, pero no lo consigue y, en el fracaso de esta trascendencia, en un segundo momento, se demora en la superficie del cuadro, disfruta de 
las puras impresiones sensibles ${ }^{29}$. Constantemente se produce este cruce de miradas: los aspectos sensibles que percibimos sobre la superficie se nos imponen y se hacen respetar y las queremos recuperar, repetir, se manera que su determinación objetiva se vuelve provisional y nos reenvía a la inspección de la superficie.

Esta explicación nos permite entender el estatuto fenomenológico de toda pintura (sea figurativa o no) y el hecho de que la experiencia estética coincide con la fenomenológica. Cada una con sus propios medios, pintura y fenomenología, motivan la misma actitud. En efecto, la actitud fenomenológica se define por su interés en el aparecer de los objetos más que por los objetos mismos. En la actitud natural de la práctica cotidiana estamos inmersos en los objetos y vivimos sus apariciones solo "a través" sin llegar a verlas. Para entrar en la actitud fenomenológica hay que distanciarse de la actitud natural. Para tomar bien esta distancia y asegurarla, Husserl introduce el concepto de epokhé con el que se pone entre paréntesis la actitud natural. Entonces neutralizamos la tendencia natural a fijar conceptualmente lo que se nos presenta. Una vez neutralizada esta tendencia, los diferentes aspectos sensibles que eran vividos pero no tematizados, solo vistos "a través", pasados por alto y dejados atrás, entran en el foco de nuestra atención y aparece también la diferencia entre el juego de apariciones sensibles y el objeto, entonces no nos interesamos por el objeto, sino por el objeto en su aparecer. La tensión entre los dos estratos de la pintura llevada a cabo por el impresionismo nos permite entender en términos pictóricos la epokhé de Husserl como la neutralización de la intención natural hacia el objeto percibido y su reducción en la superficie del cuadro.

El impresionismo produjo el giro estético del arte y mostró retrospectivamente la esencia estética de todo arte. Dicho en otros términos, fue solo a partir del arte moderno que se asumió la conciencia de que el estrato de los múltiples aspectos sensibles -el estrato estético-, siempre había estado ahí, como parte esencial de toda pintura, aunque de modo no temático, desapercibido en cuanto arte figurativo. Cuando la parte estética de la pintura se puso en primer término, se reconsideró tanto el pasado como el futuro de la pintura. Con el pasado se estableció una unidad estética, se mostró que la unidad de la pintu-

${ }^{29}$ Gehlen, Arnold. Imágenes de época. Sociología y estética de la pintura moderna. Barcelona: Península, 1994, pp. 103, 104. 
ra, a pesar de la pluralidad histórica de estilos, era una unidad estética. Con respecto al futuro se abrió la vía a la abstracción en la que no se representó ya ningún objeto mundano, desapareció la apariencia del objeto y apareció inevitablemente una pintura sin objeto, una pura superficie pictórica, como por ejemplo los cuadros de Kandinsky, de Malevich y de Mondrian. Y, finalmente, también se abrió paso al ready-made de Duchamp pues desde el momento en que el objeto se puso entre paréntesis mediante una epokhé que nos remite a su aparecer estético, entonces sobre cualquier objeto se podía practicar esa epokhé, como hizo Duchamp exhibiendo un urinario en una galería de arte.

\section{CONCLUSIÓN}

Otra interpretación del proceso histórico que lleva a la pintura abstracta es posible: la que afirma que con dicha abstracción el arte llega a su fin del arte. Esta es la interpretación de Danto que ahora vamos a considerar críticamente. Según esta interpretación, en vez de afirmar el valor estético de la pintura moderna en continuidad con toda su historia, se interpreta la abstracción (la expulsión del objeto representado en el cuadro) como la muerte de la pintura. La abstracción pictórica da pie a interpretar el arte en los términos de Hegel y de Danto pues en este tipo de arte se consuma la separación entre los dos estratos constitutivos de la pintura. El hecho de que la pura superficie estética no remita a ningún objeto, se puede interpretar en el sentido de una carencia de lo estético para alcanzar a decir algo: lo estético se revela como insuficiente, demasiado poco, y hay que recurrir, como dice Danto, a la filosofía para explicar lo que los sentidos no alcanzan. Una pintura sin el estrato del objeto se vuelve muda, cuando el objeto es expulsado del cuadro también, necesariamente, el concepto, es decir, lo que podemos reconocer y decir. Es entonces cuando el concepto se instala junto a la obra en forma de comentario, crítica o manifiesto $^{30}$. Cuando el arte se ha despedido del objeto (del concepto, del sentido) necesita una nueva legitimación que, según Danto, como hemos visto, ya no puede ser estética, sino filosófica.

\footnotetext{
${ }^{30}$ Gehlen, A. Op. Cit., p. 259. "Cuando la visión se dirige a sí misma, las formas y colores adquieren valor por sí mismos y se pierde el "sentido del objeto"; entonces la mudez entra en el cuadro. Cuando la mirada inmanente empieza a perder el sentido del objeto se está dando el primer paso hacia la decisión de suprimir de manera completa el objeto y hacer una pintura no figurativa."
} 
Según la teoría del arte de Danto, el contenido de las obras de arte es fundamentalmente diferente del aspecto que muestran los sentidos. Debe superarse el nivel estético para llegar al sentido. Las obras encarnan ideas que son invisibles en las obras y que son atribuidas por el artista, los espectadores, etc. El sentido del arte se encuentra fuera de las obras, en los comentarios que las acompañan y, en general, en todo el entorno del arte (Artworld). Ya no se trata de disfrutar estéticamente del arte, sino de entenderlo filosóficamente pues la visión, por sí misma, no alcanza ninguna objetividad y, por lo tanto, requiere una explicación desde fuera del ejercicio mismo de la visión.

Pero es justamente en la pretensión de buscar explicaciones fuera de la visión donde se muestra la confusión de Danto entre percepción estética y percepción sensible cotidiana. Esta última recurre constantemente a conceptos exteriores a ella para explicar (simplificar y abreviar) lo que la misma percepción da, pero esto es justamente lo que la percepción estética nunca hace aunque, como en los casos de la pintura abstracta, no parece que la percepción pueda llagar por sí misma a ningún concepto o sentido determinado. Pero buscar alguna explicación exterior para dar cuenta del cuadro abstracto, implica renunciar al reto estético que pone la obra al espectador. Dicho de otro modo: recurrir a la filosofía para superar la estética significa renunciar a comprender el arte en sus propios términos.

Desde la actitud natural de la percepción ciertamente nada distingue, siguiendo el ejemplo que pone Danto, la Brillo Box de una caja de detergente o, añadimos, Fountain de un urinario. La percepción sensible que es suficiente para un objeto no artístico no lo es parar la percepción de una obra de arte ${ }^{31}$. Dos objetos de aspecto idéntico aparecen de manera diferente si los percibimos según la actitud cotidiana o si los contemplamos estéticamente. Como hemos visto, la experiencia estética consiste en poner entre paréntesis la percepción sensible cotidiana, esto es, la explicación de la percepción, desde fuera, por un concepto. En el caso del ready-made el propio espacio del museo o de la galería, a diferencia de los espacios de la vida cotidiana, como un supermercado, ayudan a realizar esta operación de epokhé fenomenológica. Sin la distancia estética, que es una distancia explícita entre la percepción sensible y el concepto (entre el contenido de la obra y su medio de presentación sensible), estaría-

\footnotetext{
${ }^{31}$ Seel, M. op. cit., p. 186.
} 
mos simplemente ante un objeto práctico que nos pasa desapercibido en su exhibición estética porque solo lo comprendemos según su concepto práctico. Lo que no importa en los objetos cotidianos, es precisamente lo que sí importa en el arte: la configuración sensible o el medio del aparecer en el que este tipo de objeto se presenta.

La forma de aparecer de la obra de arte es diferente de cualquier otra porque todo lo que en la obra aparece lo hace mediante el medio sensible. Cualquier objeto no artístico (objeto práctico, proposición, signo) requiere también de un medio sensible, pero no se presenta a sí mismo como medio sensible o medio del aparecer. En cambio la obra de arte solo muestra algo, su significado, en la medida en que se muestra a sí misma en una configuración sensible intraducible a cualquier otra ${ }^{32}$. El medio del aparecer es insustituible para determinar lo que en cada caso aparece. La experiencia estética nos puede llevar a algún contenido conceptual e, incluso, podemos cuestionar el significado del arte en su totalidad, pero este contenido no será un conocimiento conceptual en sí mismo, porque siempre permanece ligado al aparecer sensible de la obra de arte concreta a la que este contenido está ligado. Toda percepción del arte empieza y acaba en el aparecer ${ }^{33}$. 